\title{
The Effect of Magnetic Field on Readout of Diodes Used as NIEL Counters
}

\author{
I. Mandić, F. Ravotti, Member, IEEE, M. Glaser, I. Serša, J. Hartert, S. Franz, V. Cindro, I. Dolenc, A. Gorišek, \\ G. Kramberger, and M. Mikuž
}

\begin{abstract}
Voltage measurement at a given forward current in dedicated dosimetric diodes is used to monitor displacement damage in silicon caused by energetic hadrons in the LHC experiments. The diodes operate in a strong magnetic field which influences their performance. In this work, measurements of the effect of the magnetic field on two types of devices: high sensitivity CMRP diodes and commercial BPW34F silicon $p-i-n$ diodes are presented. Our results show that the magnetic field effects are manageable and will not significantly deteriorate the performance of the displacement damage measurements.
\end{abstract}

Index Terms-Accelerators, dosimetry, magnetic field effects, $p-i-n$ diodes.

\section{INTRODUCTION}

D ETECTORS and readout electronics of the high-energy physics experiment at the Large Hadron Collider (LHC) at CERN [1], [2] will be exposed to high levels of radiation. The radiation causes damage to detector equipment so it is important to monitor the accumulated doses and particle fluences during detector operation. In the on-line radiation monitoring system of the ATLAS experiment [3], [4] $p-i-n$ diodes are used for monitoring of displacement damage in silicon. These sensors are calibrated in units of 1-MeV neutron equivalent fluence [5]. Irradiation with energetic hadrons changes the current-voltage characteristics of diodes. More specifically, the equivalent fluence is determined from the increase of the diode's forward voltage when $1 \mathrm{~mA}$ forward current is applied during a short time interval (hundreds of ms). The two types of diodes used in ATLAS are: CMRP diodes, which are high-sensitivity devices with a dynamic range from $10^{9} \mathrm{n} / \mathrm{cm}^{2}$ to $10^{12} \mathrm{n} / \mathrm{cm}^{2}$, and commercial BPW34F diodes, which cover the range of fluences from about $10^{12} \mathrm{n} / \mathrm{cm}^{2}$ to $10^{15} \mathrm{n} / \mathrm{cm}^{2}[5]$.

Manuscript received September 17, 2010; revised November 25, 2010; accepted November 29, 2010. Date of publication February 10, 2011; date of current version June 15, 2011.

I. Mandić, I. Serša, V. Cindro, I. Dolenc, A. Gorišek, and G. Kramberger are with the Jožef Stefan Institute, 1001 Ljubljana, Slovenia (e-mail: igor.mandic@ijs.si).

F. Ravotti, M. Glaser, and S. Franz are with CERN, 1211 Geneva 23, Switzerland.

J. Hartert is with Physikalisches Institut Universität Freiburg, 79104 Freiburg, Germany.

M. Mikuž is with the Jožef Stefan Institute, 1001 Ljubljana, Slovenia, and also with the Faculty of Mathematics and Physics, University of Ljubljana, 1000 Ljubljana, Slovenia.

Color versions of one or more of the figures in this paper are available online at http://ieeexplore.iee.org.

Digital Object Identifier 10.1109/TNS.2010.2097277
The basic unit of the monitoring system is a Radiation Monitor Sensor Board (RMSB), which hosts radiation detectors. In the innermost part of the ATLAS detector (called Inner Detector-ID [1], [6]) RMSBs are placed at 14 locations and provide on-line information of the ionizing dose in $\mathrm{SiO}_{2}$ and NonIonizing Energy Loss (NIEL) in silicon as well as estimate of thermal neutron fluence.

A simplified version of RMSBs, covering lower dose ranges, are installed at 48 locations outside of the Inner Detector, i.e., at larger distances from the interaction point, near radiationsensitive electronic equipment.

In high-energy physics experiments a magnetic field is used to bend trajectories of charged particles enabling measurements of their momenta. The ATLAS detector uses two large superconducting magnet systems [1]: the solenoid with about $1.2 \mathrm{~m}$ radius and $7 \mathrm{~m}$ in length produces a uniform magnetic field of $B=2 \mathrm{~T}$ surrounding the Inner Detector. The outer toroidal magnetic field is produced by eight very large $(25 \mathrm{~m}$ long, $5 \mathrm{~m}$ wide) air-core superconducting barrel loops and two end-caps. The toroidal field is non-uniform, with an average value of about $0.5 \mathrm{~T}$ in the barrel region. Therefore, almost the entire detector system together with radiation monitors operates in a relatively high magnetic field.

During the starting run of the ATLAS experiment, it was discovered that the performance of radiation monitor $p-i-n$ diodes is influenced by the magnetic field. The readout voltage of a radiation monitoring diode (CMRP) significantly increases when the magnetic field is turned on. No significant changes of outputs of other radiation sensors (e.g., RadFET devices as described in [5]) hosted by RMSBs were observed when magnetic field was switched on and off. Fig. 1 shows the change of forward voltage when magnetic field is on, relative to the value with no field, at $1 \mathrm{~mA}$ forward current in two types of diodes at 14 locations in the Inner detector. Of the 14 monitoring locations, 6 are in the central barrel [4] where magnetic field is $2 \mathrm{~T}$ while 8 locations are on the Inner Detector end plate which is outside of the region with uniform solenoid field [2]. The field value at these locations is less than $0.5 \mathrm{~T}$ [7]. It can be seen that the magnetic field effect is significantly larger in the region of high field.

Following this observation, a measurement campaign was started in order to quantify the magnetic field effect on dosimetric $p-i-n$ diodes used in our system. The magnetic field is present in the detector most of its operation time so the effect of the field must be known to enable on-line radiation monitoring. The magnitude of the effect could be measured when the field is switched off during technical stops scheduled every several weeks. But the concern for the radiation monitors was the 

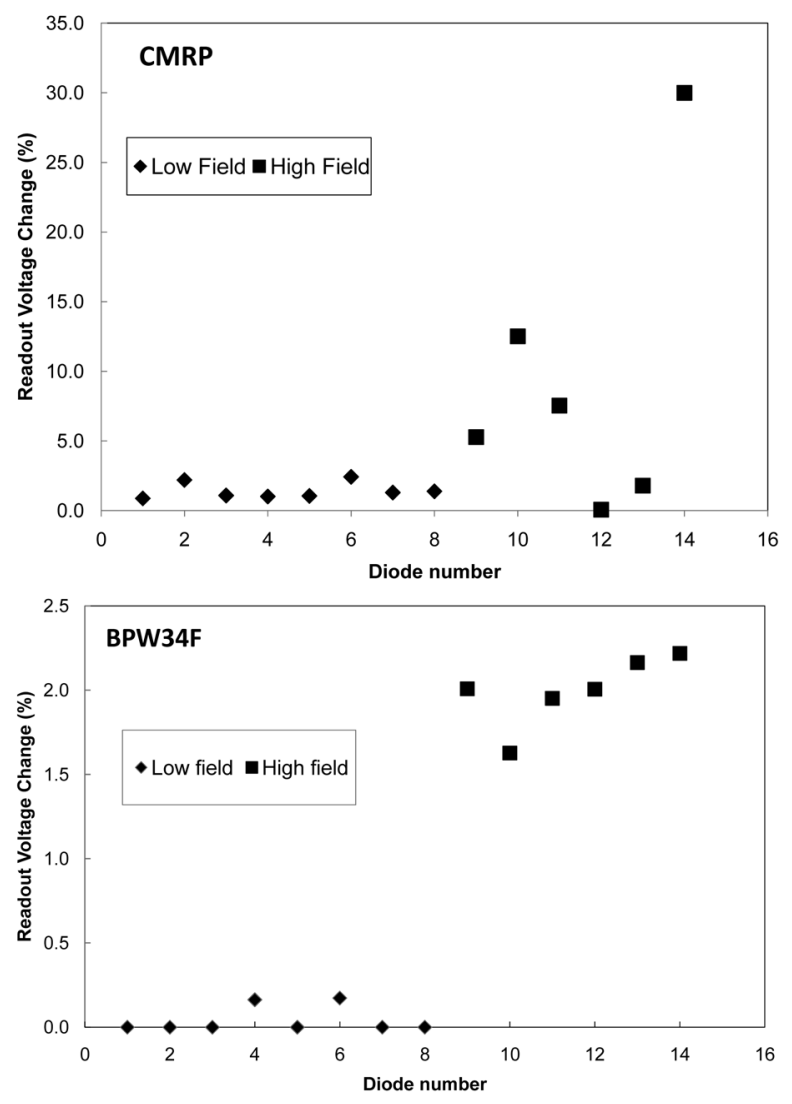

Fig. 1. Relative readout voltage change when the solenoid magnetic field is turned on for diodes at 14 monitoring locations in the Inner Detector. Points marked with "High field" are in locations in the Inner Detector barrel where $B=2 \mathrm{~T}$ while "Low field" are monitoring locations on the Inner Detector end plate where magnetic field is lower $(\sim 0.5 \mathrm{~T})$.

dependence of this effect on the NIEL accumulated by the sensors.

\section{MEASUREMENTS}

It is known that the resistivity of silicon may increase significantly under strong magnetic field due to the so called magneto-resistance effect. If the magnetic field is not parallel to the direction of electrical current, the Lorentz force acts on charge carriers. In a slab of silicon, where the current is carried by two types of carriers, the consequence of the Lorenz force is increased resistance. The ratio of increased resistivity to the resistivity at zero magnetic field is proportional to the square of magnetic field component perpendicular to the direction of the current flow [8].

The change of forward voltage characteristics in diodes due to the magnetic field has been observed long ago. One of early measurements can be found in [9]. The effect is exploited in magnetic field sensors called magnetodiodes. In [10] the qualitative explanation of the effect is given and in [11], [12] a detailed theoretical treatment of the influence of a magnetic field on the current-voltage characteristics can be found. In general, in a $p-i-n$ diode operated in forward bias, the current in the intrinsic $i$ part is carried by both types of carriers, which are injected into it from the $p+$ and $n+$ sides. In the presence of magnetic field the carriers are pushed by the Lorenz force. In addi- tion to already mentioned magneto-resistance effect, the consequence of the Lorenz force is also that carriers move nearer to the edges of the device where the recombination probability is higher. The resulting change of forward current-voltage characteristic depends on large number of parameters such as the dimensions of the device, its surface properties, carrier injection level etc. The increase of forward voltage at given forward current in magnetic field observed in diodes used in our system may be attributed to magneto-resistance as well as to the increased carrier recombination.

The effect of the magnetic field on both CMRP and BPW34F diodes was studied by series of experiments performed with two different magnets: the superconducting magnet of the Jožef Stefan Institute (JSI magnet in the following) and in the magnet available at one of the beam lines of the CERN PS (CERN magnet in the following).

The JSI superconducting magnet provides a homogeneous magnetic field of $B=2.35 \mathrm{~T}$ over a volume of at least $10 \times 10 \times 10 \mathrm{~cm}^{3}$. The CERN magnet can be operated with a current, which can be set from 0 to $2300 \mathrm{~A}$. This creates a uniform magnetic field over a volume of several $\mathrm{cm}^{3}$ with intensity up to $1.37 \mathrm{~T}$ for the maximum available current. The accuracy and reproducibility of the field at each step of the measurement were well below $1 \%$.

Diodes were characterized by measuring the forward voltage at $1 \mathrm{~mA}$ forward current following the standard readout protocol [3]. Measurements were done at different diode's orientations with respect to the magnetic field direction and at different values of magnetic field. Temperature variations between measurements were taken into account by scaling the readout values to $20^{\circ} \mathrm{C}$ using known temperature dependences [5].

It has been found that the magnetic field effect depends on only one angle between the diode package and the direction of the magnetic field. The relevant angle for both types of diodes is defined on the drawing in Fig. 2. The effect does not dependent on the polarity of magnetic field i.e., $180^{\circ}$ change of direction of magnetic field doesn't change the readout values.

The diodes were irradiated with neutrons in the TRIGA reactor of Jožef Stefan Institute in Ljubljana [13], [14] while irradiations with $23 \mathrm{GeV}$ protons were performed in IRRAD facility at CERN PS accelerator [15].

\section{A. CMRP Diodes}

CMRP are the high sensitivity diodes produced at the Center for Medical Radiation Physics (CMRP) of the university of Wollongong, Australia. They are ion implanted $p-i-n$ diodes made of high resistivity silicon $(\rho \sim 10 \mathrm{k} \Omega \cdot \mathrm{cm})$ [5], [16] with intrinsic part $(i)$ length of $1 \mathrm{~mm}$ and a cross section of $p+$ and $n+$ implants of $1.2 \mathrm{~mm}^{2}$ at each side of the bulk. Measurements of relative readout voltage increase in $2.35 \mathrm{~T}$ magnetic field in the JSI magnet at different angles $\vartheta$ made before and after irradiation are shown in Fig. 3. The change of forward voltages at $1 \mathrm{~mA}$ current pulse relative to the value outside of magnet is shown for various diodes as a function of angle $\vartheta$ (as defined in Fig. 2). It can be seen that the magnetic field effect is significant and can be up to $90 \%$, that it depends on the angle $\vartheta$ and varies substantially from device to device. As already mentioned rotations of 


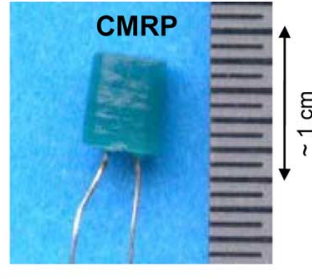

(a)

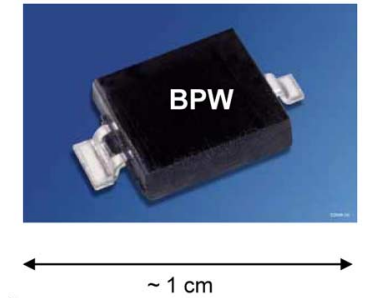

)
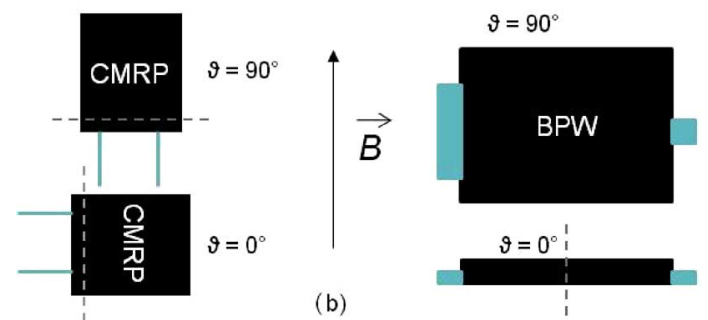

Fig. 2. (a) Photos of the two diode types. (b) definitions of angle $\vartheta$. For CMRP $\vartheta$ is the angle between the direction of magnetic field and the line joining the two contacts on the diode package while for BPW the angle $\vartheta$ is between the direction of magnetic field and the direction normal to the surface of the package.

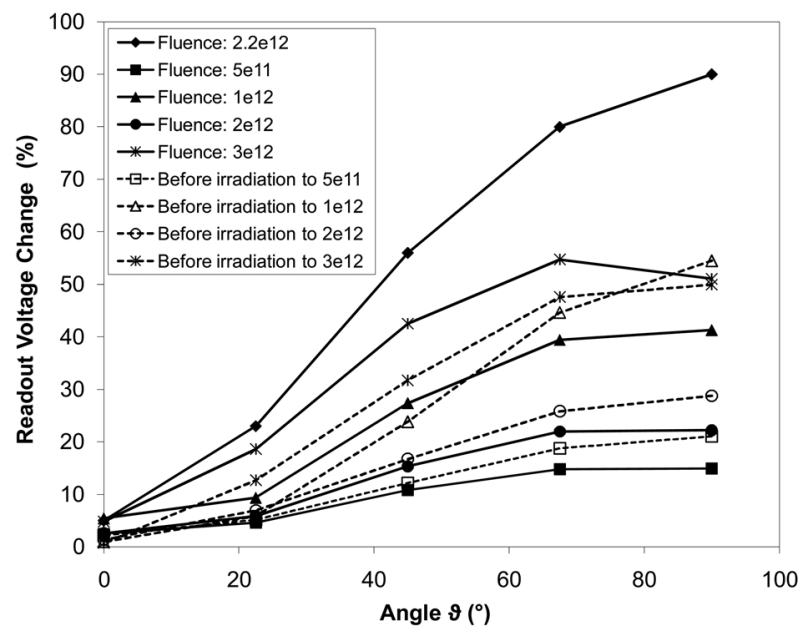

Fig. 3. Relative change of CMRP voltage vs. angle between the direction of the magnetic field $(\mathrm{B}=2.35 \mathrm{~T}$ ) and diode before and after irradiation. The fluences are in units of $1 \mathrm{MeV}$ eq. neutrons per $\mathrm{cm}^{2}$. Please note that for the diode irradiated with protons to equivalent fluence of $2.2 \cdot 10^{12} \mathrm{n} / \mathrm{cm}^{2}$ the measurement before irradiation is not available.

diodes around the axis defined by the direction of magnetic field don't change the readout voltage.

It can also be seen in Fig. 3 that the relative change of readout voltage doesn't depend strongly on irradiation fluence. For example: if the increase of the readout voltage due to the magnetic field at certain angle is $\sim 20 \%$ before irradiation it will be about $20 \%$ also after irradiation. This is an important finding as it enables a simple correction of magnetic field effects in radiation monitor application. At angle $\vartheta \sim 0$ the effect of magnetic field before and after irradiation is about $5 \%$ or smaller which is well below the precision of dose measurements. In Fig. 4 measurement of readout voltage change as a function of field is shown for one un-irradiated device. It can be seen that the relative readout voltage change increases with magnetic field.

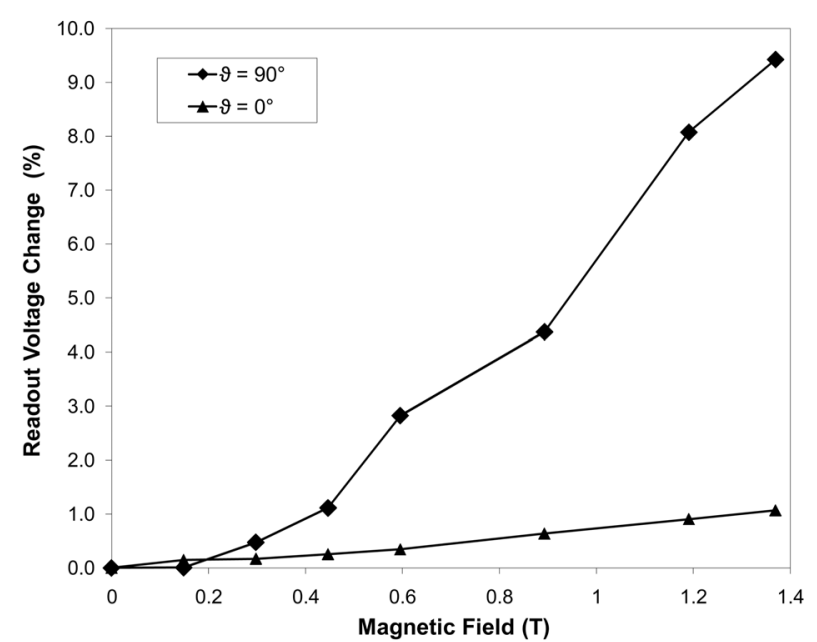

Fig. 4. Relative change of readout voltage in CMRP diode as a function of magnetic field strength. The diode was not irradiated.

A more precise characterization of the magnetic field effect would require a better knowledge of the exact orientation of the die inside the plastic package of the CMRP.

\section{B. BPW34F Diodes}

BPW34F are commercial silicon $p-i$ - $n$ diodes produced by several companies and used in many applications such as IR remote controls. They are processed from silicon with resistivity $\rho \sim 2.5 \mathrm{k} \Omega \cdot \mathrm{cm}$. The diode's base thickness is $W=210 \mu \mathrm{m}$, and active surface is $\sim 2.65 \times 2.65 \mathrm{~mm}^{2}$ [17]. The packaging of the diode and the definition of angle $\vartheta$ is shown in Fig. 2. Diodes used in ATLAS radiation monitor were produced by OSRAM [18]. They were pre-irradiated with $\Phi_{\mathrm{eq}}=3 \cdot 10^{12} \mathrm{n} / \mathrm{cm}^{2}$ with reactor neutrons to extend their sensitivity to low fluence range [4], [5], [17].

In Fig. 5 measurements of relative readout voltage change in two irradiated BPW34F diodes as a function of magnetic field at three different angles $\vartheta\left(0,45\right.$ and $\left.90^{\circ}\right)$ are shown. Fig. 6 shows measurements of the effect of field versus the square of magnetic field made at angle $\vartheta=90^{\circ}$ for six diodes, four irradiated with reactor neutrons and two with $23 \mathrm{GeV}$ protons. It can be seen from Figs. 5 and 6 that the effect of field, different than in the case of CMRP diodes, increases with irradiation fluence. Fig. 7 shows the effect of field $B=2.35 \mathrm{~T}$ as a function of equivalent fluence measured at three different angles $\vartheta$. It can be seen that the effect of field saturates at fluences higher than $\sim 1.5 \cdot 10^{13} \mathrm{n} / \mathrm{cm}^{2}$. Points at each fluence in Fig. 7 represent measurements with different diode indicating small device-to-device variations. Results obtained with proton and neutron irradiated diodes are shown. Good agreement between the two sets can be seen.

The effect of $B=2.35 \mathrm{~T}$ at angle $\vartheta=90^{\circ}$ was measured also on a set of 7 pre-irradiated BPW34F diodes (i.e., irradiated to $\Phi_{\text {eq }}=3 \cdot 10^{12} \mathrm{n} / \mathrm{cm}^{2}$ ). The average relative voltage increase was found to be $3.5 \%$ with a spread of $0.3 \%$ confirming the small device-to-device variations, opposite to the case of CMRP diodes where variations are significant. However, one can note that the effect for the diode at lowest fluence in Fig. 7 is larger than $3.5 \%$. This discrepancy seen in this particular case could 

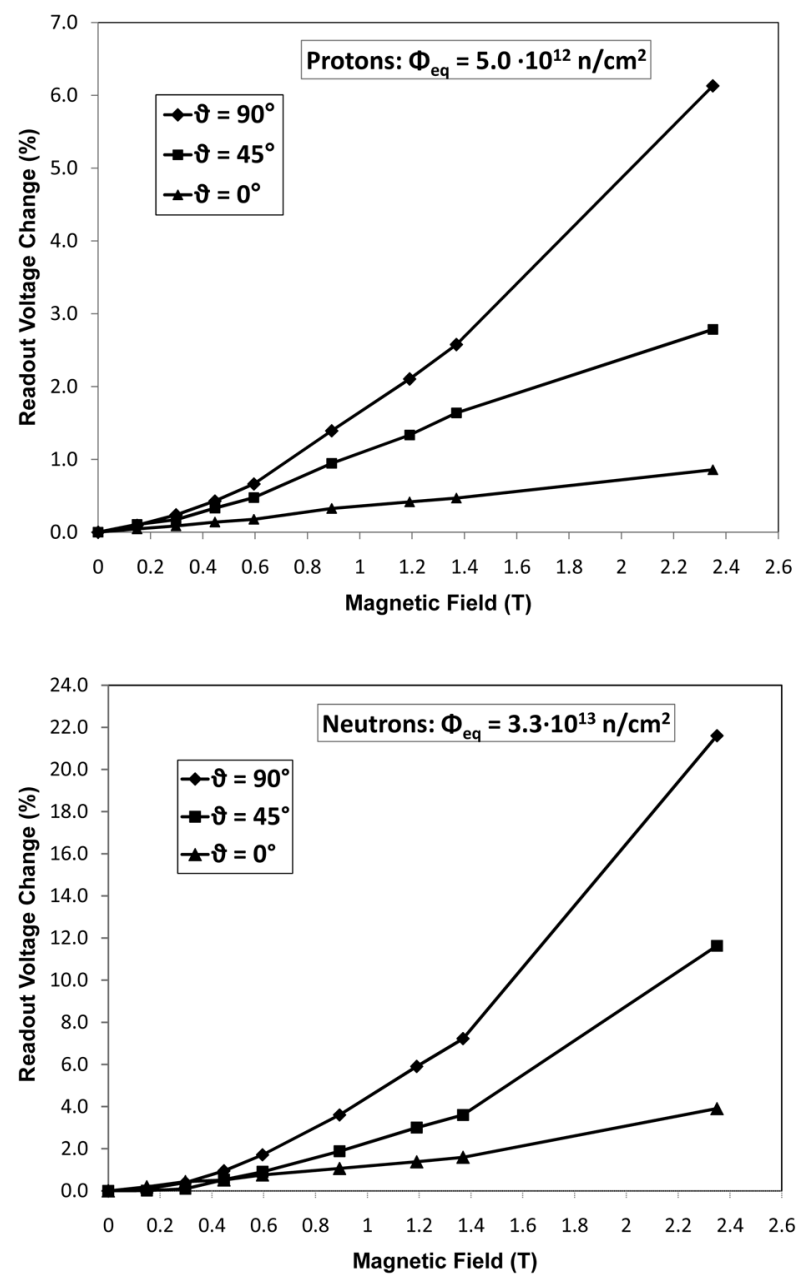

Fig. 5. Relative change of readout voltage in two irradiated BPW34F diodes as a function of magnetic field at three different angles $\vartheta$.

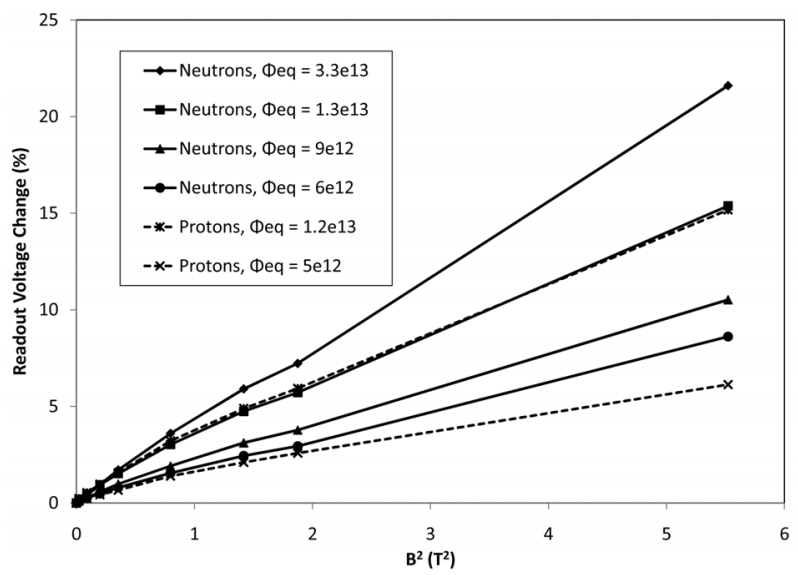

Fig. 6. Relative change of readout voltage in irradiated BPW34F diodes as a function of square of magnetic field at angle $\vartheta=90^{\circ}$ for diodes irradiated to different fluences with neutrons and protons.

be a fluctuation and could also be attributed to the error in irradiation fluence, temperature corrections etc.

By scaling linearly with $B^{2}$ (see Fig. 6) the expected magnetic field effect at $B=2 \mathrm{~T}$ and $\vartheta=90^{\circ}$ for fluence $\Phi_{\mathrm{eq}}=3$. $10^{12} \mathrm{n} / \mathrm{cm}^{2}$ would be $2.5 \%$ if it is $3.5 \%$ at $B=2.35$ T. In Fig. 1

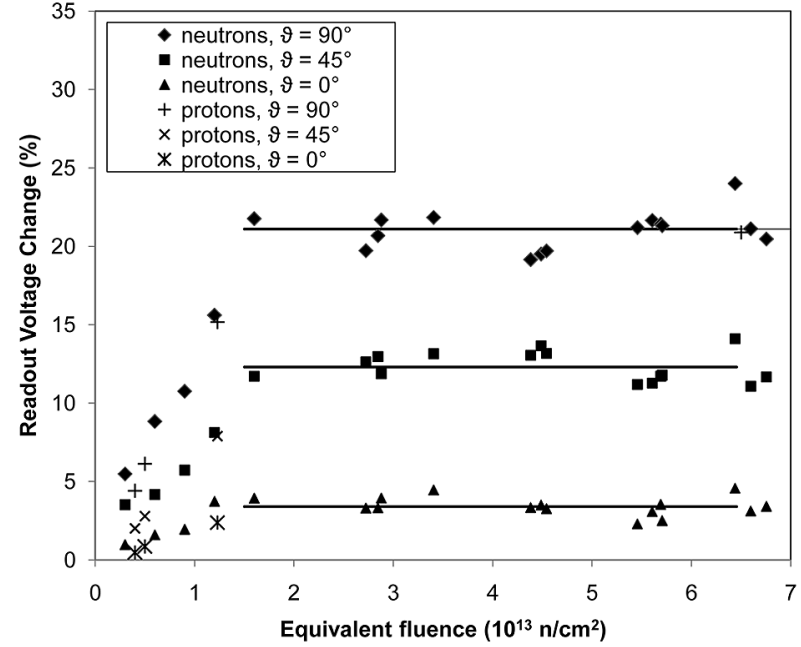

Fig. 7. Effect of magnetic field $B=2.35 \mathrm{~T}$ on readout voltage in BPW34F diodes irradiated with reactor neutrons and $23 \mathrm{GeV}$ protons as a function of 1 $\mathrm{MeV}$ equivalent neutron fluence measured at three different angles $\vartheta$. Horizontal lines are averages of relative voltage change at fluences higher than $1.5 \cdot 10^{13}$ $\mathrm{n} / \mathrm{cm}^{2}$. Each point in the figure represents a measurement made on different diode.

it can be seen that the effect measured in ATLAS where pre-irradiated diodes are used for "High field" (i.e., $B=2 \mathrm{~T}$ and $\vartheta \sim 90^{\circ}$ ) diodes is $\sim 2 \%$. This is satisfactory agreement with measurements in JSI magnet, taking into account various differences between laboratory measurement setup and the readout system of the ATLAS radiation monitor, uncertainties of diode orientations etc.

With BPW34F diodes the fluence is calculated from $\Phi=$ $k \cdot \Delta V$ with $k=9.1 \cdot 10^{12} \mathrm{n} /\left(\mathrm{cm}^{2} \mathrm{~V}\right)$. The expected initial voltage increase of $2.5 \%(\sim 16 \mathrm{mV})$ due to the magnetic field is therefore similar to the effect of $1 \mathrm{MeV}$ neutron fluence of about $\sim 2 \cdot 10^{11} \mathrm{n} / \mathrm{cm}^{2}$ which is below sensitivity of this diode and can be neglected in case of application in ATLAS radiation monitor. But as the dose in the experiment is accumulated the effect starts to be significant and should be taken into account. The effect increases with fluence until it reaches saturation value of $\sim 16 \%$ at about $\Phi_{\text {eq }} \sim 1.5 \cdot 10^{13} \mathrm{n} / \mathrm{cm}^{2}$.

\section{CONCLUSION}

In this work, the effect of magnetic field on the readout voltage of dosimetric diodes is reported. An increase of the bias voltage needed for $1 \mathrm{~mA}$ forward current induced by the uniform magnetic field with density up to $2.35 \mathrm{~T}$ was measured.

The measurements were done with two types of diodes used for NIEL measurements in radiation monitoring application: CMRP and BPW34F. Table I summarizes the main characteristics of magnetic field effect for the two diode types.

Measurements with CMRP showed large spread of the effect, ranging from less than $20 \%$ to up to $90 \%$. The effect depends on the orientation of diodes relative to the magnetic field. The effect does not change significantly after diodes are irradiated. For the application in the ATLAS radiation monitor, the effect should be quantified for each individual diode by comparing the readout voltages with the magnetic field on and off. 
TABLE I

SUMMARY OF THE MAGNETIC FIELD EFFECT

\begin{tabular}{|c|c|c|}
\hline & $\begin{array}{c}\text { CMRP } \\
(B=2 \mathrm{~T})\end{array}$ & $\begin{array}{l}\text { BPW34F } \\
(B=2 \mathrm{~T})\end{array}$ \\
\hline $\begin{array}{c}\text { Initial } \\
\text { correction }\end{array}$ & $\begin{array}{c}10 \% \text { - } 90 \% \text {, large } \\
\text { device to device } \\
\text { variations }\end{array}$ & $\begin{array}{c}2 \%, \\
\text { small device to device } \\
\text { variations }\end{array}$ \\
\hline $\begin{array}{c}\text { Dependence } \\
\text { on } \\
\text { orientation }\end{array}$ & Yes & Yes \\
\hline $\begin{array}{c}\text { Change with } \\
\text { irradiation }\end{array}$ & No change & $\begin{array}{c}\text { Linear increase until } \\
\Phi_{e q} \sim 1.5 \cdot 10^{13} \mathrm{n} / \mathrm{cm}^{2}, \\
\text { no change at higher } \\
\text { fluence }\end{array}$ \\
\hline $\begin{array}{l}\text { Maximal } \\
\text { expected } \\
\text { effect }\end{array}$ & $0 \%-90 \%$ & $\begin{array}{c}16 \% \\
\text { when } \Phi_{e q}>1.5 \cdot 10^{13} \\
\mathrm{n} / \mathrm{cm}^{2}\end{array}$ \\
\hline $\begin{array}{c}\text { Scaling with } \\
\text { B }\end{array}$ & $B^{2}$ & $B^{2}$ \\
\hline
\end{tabular}

In the case of BPW34F diodes the effect depends on diode's orientation but it doesn't change significantly from device to device and it depends on irradiation fluence as can be seen in Figs. 5 and 6. At low fluences $\Phi_{\text {eq }}<5 \cdot 10^{12} \mathrm{n} / \mathrm{cm}^{2}$ the effect is small and may be neglected in ATLAS radiation monitoring application. At fluences higher than $\Phi_{\text {eq }} \sim 1.5 \cdot 10^{13} \mathrm{n} / \mathrm{cm}^{2}$ the effect doesn't change with fluence therefore same correction constant may be used after this fluence is accumulated. In the intermediate fluence range the correction constant should be determined when the magnetic field is turned off during maintenance intervals which are scheduled every few weeks. From Figs. 6, 7 and 8 it follows that the effect of magnetic field on BPW34F diodes will be less than $16 \%$ during the whole lifetime of ATLAS experiment.

The influence of magnetic field to forward current-voltage characteristics of $p-i-n$ diodes may be caused by several effects. One of them is the magneto-resistance which causes the increase of ohmic resistance of the long base in $p-i-n$ diode resulting in increase of voltage at given forward current. The magnetoresistance effect is proportional to $\sim B^{2}$. The curves of relative change of readout voltage with magnetic field shown in Fig. 4 (CMRP diodes) and Fig. 5 (BPW34F diodes) have the concave shape and can be well fitted with second order polynomial. Fig. 6 clearly shows that the voltage increase is proportional to the square of magnetic field. Such scaling is expected if increase of forward voltage is caused by increased resistance in the diode's base due to the magneto-resistance.

The sensitivity of $p-i$ - $n$ diodes to neutrons depends on material resistivity and base length (i.e., length of $i$ part). The high sensitivity CMRP diodes are made of high resistivity material with long base. Their response to neutrons is dominated by the increase of resistivity of material while the modulation of base length is not significantly affected by the reduction of carrier life time. This may also be the reason for weak dependence of their response to magnetic field on irradiation fluence since the mag- netic field effect is the consequence of magnetoresistivity in the diodes's base which does not change due to irradiation.

In the case of BPW34F diodes the neutron sensitivity at low fluences is affected by the carrier lifetime reduction which leads to increase of the length of not-modulated part of the diode's base. This causes also the increase of magnetoresistivity effect which saturates when the carrier life time becomes too small to significantly modulate the base as the fluence is increased.

\section{ACKNOWLEDGMENT}

The authors would like to thank the crews of the TRIGA reactor in Ljubljana and the operators of the PS accelerator at CERN for their help with irradiations and tests with experimental magnets.

\section{REFERENCES}

[1] G. A. Aad et al., The ATLAS collaboration, "The ATLAS experiment at the CERN large hadron collider," JINST, 2008, JINST 3 S08003.

[2] ATLAS Detector and Physics Performance Tech. Design Rep., CERN/ LHCC/99-14/15, 1999.

[3] I. Mandić, V. Cindro, A. Gorišek, G. Kramberger, and M. Mikuž, “Online integrating radiation monitoring system for the ATLAS detector at the large hadron collider,' IEEE Trans. Nucl. Sci., vol. 54, no. 4, pp. 1143-1150, Aug. 2007.

[4] J. Hartert, J. Bronner, V. Cindro, A. Gorišek, I. Mandić, and M. Mikuž, "The ATLAS radiation dose measurement system and its extension to SLHC experiments," in Proc. Topical Workshop Electronics for Particle Physics, Naxos, Greece, Sep. 15-19, 2008 [Online]. Available: http://indico.cern.ch/getFile.py/access?contribId=121\&sessionId=15\&resId=0\&materialId=paper\&confId $=21985$

[5] F. Ravotti, M. Glaser, and M. Moll, SENSOR catalogue-Data compilation of solid-state sensors for radiation monitoring, CERN TS Note, 2005-002, May 13, 2005.

[6] ATLAS TDR, Inner Detector Tech. Design Rep., CERN/LHCC/97-17, 1997.

[7] M. Aleksa, F. Bergsma, L. Chevalier, P.-A. Guidici, J. C. Hart, A. Kehrli, M. Losasso, P. S. Miyagawa, X. Pons, H. Sandekar, and S. W. Snow, "Results of the ATLAS solenoid magnetic field map," in J. Phys., Conf. Series, 2008, vol. 110, p. 092018, DOI:10.1088/17426596/110/9/092018.

[8] B. Sapoval and C. Hermann, Physics of Semiconductors. New York: Springer-Verlag, 1995.

[9] R. V. Aldridge et al., J. Phys. D, Appl. Phys., vol. 8, p. 64, 1975.

[10] J. Lenz and S. Edelstein, "Magnetic sensors and their applications," IEEE Sensors J., vol. 6, pp. 631-649, 2006.

[11] S. Cristoloveanu, "Magnetic field and surface influences on double injection phenomena in semiconductors I," Phys. Stat. Sol. (A), vol. 64, pp. 683-292, 1981.

[12] S. Cristoloveanu, "Magnetic field and surface influences on double injection phenomena in semiconductors II," Phys. Stat. Sol. (A), vol. 65, pp. 281-292, 1981.

[13] M. Ravnik and R. Jeraj, "Research reactor benchmarks," Nucl. Sci. Eng., vol. 145, pp. 145-152, 2003.

[14] L. Snoj, A. Trkov, R. Jaćimović, P. Rogan, G. Žerovnik, and M. Ravnik, "Analysis of neutron flux distribution for the validation of computational methods for the optimization of research reactor utilization," Appl. Radiat. Isot., vol. 69, pp. 136-141, 2011.

[15] M. Glaser, L. Durieu, F. Lemeilleur, M. Tavlet, C. Leroy, and P. Roy, "New irradiation zones at the CERN-PS," Nucl. Instrum. Methods Phys. Res A, vol. A426, pp. 72-77, 1999.

[16] A. B. Rosenfeld, M. Yudelev, M. Lerch, I. Cornelius, P. Griffin, V. Pervertailo, I. Anokhin, O. Zintes, V. Khivrich, M. Pinkovaskaya, D. Alexiev, and M. Reinhard, "Neutron dosimetry with planar silicon p-i-n diodes," IEEE Trans. Nucl. Sci., vol. 50, no. 6, pp. 2367-2382, Dec. 2003.

[17] F. Ravotti, M. Glaser, M. Moll, and F. Saigne, "BPW34 commercial p-i-n diodes for high-level $1-\mathrm{MeV}$ neutron equivalent fluence monitoring," IEEE Trans. Nucl. Sci., vol. 55, no. 4, pp. 2133-2140, Aug. 2008.

[18] BPW34 Silicon p-i-n Photodiode Datasheet, OSRAM OptoSemiconductors [Online]. Available: http://www.osram-os.com 